\title{
Lack of Screening in the Continuous Dipole Systems *
}

\author{
Yong Moon Park
}

Departments of Physics and Mathematics, Princeton University, Princeton, New Jersey 08544, USA

\begin{abstract}
We study continuous statistical systems interacting via a regularized dipole potential in the grand canonical ensemble. In the explicitly given region of high temperature (or low density) we show that the effective potential between two parallel dipoles is not absolutely integrable (it is, however, square integrable), which implies that the effective potential does not fall off faster than $|x|^{-3}$ in some directions.
\end{abstract}

We consider continuous statistical systems interaction via a dipole potertial in the grand canonical ensemble. Since the dipole potential is not stable, we regularize the potential at the origin so that the system is well-defined. In [3] we have shown that the thermodynamic limit of correlation functions and the pressure exists. It is of great interest to know whether there exists some kind of screening. By defining an effective potential between two dipole moments in the grand canonical ensemble it turns out that the effective potential between two parallel dipoles is not absolutely integrable in the explicitly given region of high temperature (or low density). On the other hand, we will show that it is square integrable. The result implies that the effective potential does not fall off faster than $|x|^{-3}$ in some directions (for high temperatures or low densities). This is in contrast to dilute coulomb gas for which Debye exponential screening occurs [1].

Since the dipole potential is of long-range, the method of the cluster expansion [7] cannot be applied. The technique used in the proof is the Gaussian integral formalism of statistical mechanics, which has already proved to be a very powerful technique $[2,3,4,6]$. We primarily follow the notation of [3] and use some of its results.

We first briefly introduce the notation and the model we are interested in. The regularized dipole potential between a particle with dipole moment $\sigma \in \mathbb{R}^{3}$, at position $x \in \mathbb{R}^{3}$ and one with dipole moment $\sigma^{\prime}$ at position $x^{\prime}$ is defined by

$$
V\left(\sigma, x ; \sigma^{\prime}, x^{\prime}\right)=(2 \pi)^{-3 / 2} \int d^{3} k e^{i k \cdot\left(x-x^{\prime}\right)}(\sigma \cdot k)\left(\sigma^{\prime} \cdot k\right) k^{-2}|\hat{\kappa}(k)|^{2}
$$

where $\hat{\kappa}(k)$ is the Fourier transform of a regularization function $\kappa(x)$ in $C_{0}^{\infty}\left(\mathbb{R}^{3}\right)$

* Research supported in part by NSF PHY 76-80958

$\star \star$ Permanent address: Department of Mathematics, Yonsei University, Seoul, Korea 
which satisfies the following conditions:

$$
\begin{aligned}
& \kappa(x)=\kappa(|x|), \quad \int d^{3} x \kappa(x)=1, \\
& \kappa(x)=0 \text { if }|x|>R \text { for some } R>0 .
\end{aligned}
$$

Since the function $k(x)$ is isotropic, one may apply Newton's law to see that for $\left|x-x^{\prime}\right|>2 R$ the regularized potential equals the usual dipole potential. Furthermore $\left|V\left(\sigma, x ; \sigma^{\prime}, x^{\prime}\right)\right|<\infty$ for all $\sigma, \sigma^{\prime}, x$ and $x^{\prime}$. We set

$$
\begin{aligned}
& (\sigma)_{N}=\left(\sigma_{1}, \ldots, \sigma_{N}\right), \quad(x)_{N}=\left(x_{1}, \ldots, x_{N}\right) \\
& \int_{\left(S^{2}\right)^{N}} d(\sigma)_{N} \ldots=\int\left(\prod_{j=1}^{N} d^{3} \sigma_{j} \delta\left(\left|\sigma_{j}\right|-1\right)\right) \ldots, d(x)_{N}=\prod_{j=1}^{N} d^{3} x_{j}
\end{aligned}
$$

The potential for $N$-dipole particles at position $(x)_{N}$ and with dipole moments $(\sigma)_{N}$ is given by

$$
U\left((\sigma)_{N},(x)_{N}\right)=\sum_{1 \leqq i<j \leqq N} V\left(\sigma_{i}, x_{i} ; \sigma_{j}, x_{j}\right)
$$

We also write that for $\alpha, \alpha^{\prime} \in \mathbb{R}$

$$
\begin{aligned}
& U\left(\alpha \sigma, x ; \alpha^{\prime} \sigma^{\prime}, x ;(\sigma)_{N}(x)_{N}\right)=W_{N}\left(\alpha \sigma, x ; \alpha^{\prime} \sigma^{\prime}, x^{\prime} ;(\sigma)_{N},(x)_{N}\right)+U\left((\sigma)_{N},(x)_{N}\right) \\
& W_{N}\left(\alpha \sigma, x ; \alpha^{\prime} \sigma^{\prime}, x^{\prime} ;(\sigma)_{N},(x)_{N}\right)= \alpha \alpha^{\prime} V\left(\sigma, x ; \sigma^{\prime}, x^{\prime}\right) \\
&+\alpha \sum_{j=1}^{N} V\left(\sigma, x ; \sigma_{j}, x_{j}\right)+\alpha^{\prime} \sum_{j=1}^{N} V\left(\sigma^{\prime}, x^{\prime} ; \sigma_{j}, x_{j}\right) .
\end{aligned}
$$

One may check that $W_{N}$ is the change of the potential energy when one introduces two dipoles with moments $\alpha \sigma$ and $\alpha^{\prime} \sigma^{\prime}$ at $x$ and $x^{\prime}$ respectively. For a system of unit dipoles the grand canonical partition function in a compact region $\Lambda \subset \mathbb{R}^{3}$ is defined by

$$
\mathscr{Z}_{\Lambda}(\beta, z)=\sum_{N=0}^{\infty} \frac{z^{N}}{N !} \int_{\left(S^{2}\right)^{N}} d(\sigma)_{N} \int_{\Lambda^{N}} d(x)_{N} e^{-\beta U\left((\sigma)_{N},(x)_{N}\right)}
$$

where we set the term corresponding to $N=0$ equals to one. Let

$$
\begin{aligned}
\rho_{\Lambda}\left(\beta, z ; \alpha \sigma, x ; \alpha^{\prime} \sigma^{\prime}, x^{\prime}\right)= & \mathscr{Z}_{\Lambda}(\beta, z)^{-1} \sum_{N=0}^{\infty} \frac{z^{N}}{N !} \int_{\left(S^{2}\right)^{N}} d(\sigma)_{N} \int_{\Lambda^{N}} d(x)_{N} \\
& \cdot z^{2} \exp \left[-\beta U\left(\alpha \sigma, x ; \alpha^{\prime} \sigma^{\prime}, x^{\prime} ;(\sigma)_{N},(x)_{N}\right)\right]
\end{aligned}
$$

The function $\rho_{A}$ is the two particle correlation function (distribution) with dipole moments $\alpha \sigma$ and $\alpha^{\prime} \sigma^{\prime}$ at the positions $x$ and $x^{\prime}$ respectively. We define the effective potential between a particle with dipole moment $\sigma$ at $x$ and one with dipole moment $\sigma^{\prime}$ at $x^{\prime}$ by

$$
W_{\Lambda}\left(\beta, z ; \sigma, x ; \sigma^{\prime}, x^{\prime}\right)=-\left.\frac{1}{\beta z^{2}} \frac{\partial^{2}}{\partial \alpha \partial \alpha^{\prime}} \rho_{\Lambda}\left(\beta, z ; \alpha \sigma, x, \alpha^{\prime} \sigma^{\prime}, x^{\prime}\right)\right|_{\alpha=\alpha^{\prime}=0}
$$

It is easy to check that the effective potential becomes the dipole potential (1) 
for either $z=0$ or $\beta=0$. From the results in [3] it follows that the thermodynamic limit

$$
\begin{aligned}
W\left(\beta, z ; \sigma, \sigma^{\prime} ; x^{\prime}-x\right) & =W\left(\beta, z ; \sigma, x ; \sigma^{\prime}, x^{\prime}\right) \\
& =\lim _{\Lambda \uparrow \mathbb{R}} W_{\Lambda}\left(\beta, z ; \sigma, x ; \sigma^{\prime}, x^{\prime}\right)
\end{aligned}
$$

exists and is translation invariant. We will discuss the existence of the thermodynamic limit in more detail later.

We now introduce our main result. We denote

$$
K \equiv \frac{1}{2} V(\sigma, 0 ; \sigma, 0)<\infty
$$

for $|\sigma|=1$. Our main result is:

Theorem 1. (a) For $(4 \pi / 3) z \beta e^{\beta K}<1$, the effective potential $W(\beta, z ; \sigma, \sigma ; x)$ between two parallel dipoles separated by $x \in \mathbb{R}^{3}$ is not absolutely integrable.

(b) For any $\beta \geqq 0$ and $z \in \mathbb{R}, W(\beta, z ; \sigma, \sigma ; x)$ is square integrable.

Remark. (1) Part (a) of the theorem implies that for $(4 \pi / 3) z \beta e^{\beta K}<1$ (i.e. the high temperature or low density region) exponential screening does not hold for the dipole system. In particular, it implies that effective potential does not fall off faster than $|x|^{-3}$ in some directions. The theorem (b) suggests that it falls off faster than $|x|^{-3 / 2}$ for all $\beta$ and $z$.

(2) The result is in contrast to the dilute coulomb gas for which by a slight modification of Brydges method [1] one may show that the effective potential, defined in the manner similar to that of (8) and (9), falls off exponentially.

The rest of this note is devoted to the proof of Theorem 1. As in [2] we will express the partition function and the effective potential in terms of a Gaussian integral. For details we refer the reader to [3]. Let $d \mu(\phi)$ be the Gaussian measure with mean zero and covariance given by $V\left(\sigma, x ; \sigma^{\prime}, x^{\prime}\right)$. (Notice that $V\left(\sigma, x ; \sigma^{\prime}, x^{\prime}\right)$ is of positive type.) Let $V$ be the operator on $L^{2}\left(\mathbb{R}^{3} \times \mathbb{R}^{3}\right)$ defined by its kernel $V\left(\sigma, x ; \sigma^{\prime}, x^{\prime}\right)$. For $\phi \in \mathscr{S}^{\prime}\left(\mathbb{R}^{3} \times \mathbb{R}^{3}\right)$ and $f \in \mathscr{S}\left(\mathbb{R}^{3} \times \mathbb{R}^{3}\right)$ we have

$$
\int_{\mathscr{I}^{\prime}} d \mu(\phi) e^{i \phi(f)}=e^{-1 / 2\langle f, V f\rangle}
$$

where $\langle f, g\rangle=\int d^{3} \sigma \int d^{3} x \overline{f(\sigma, x)} g(\sigma, x)$. We define Wick ordering by

$$
: e^{i \phi(f)}:=\left(\int d \mu e^{i \phi(f)}\right)^{-1} e^{i \phi(f)}
$$

From (11) and (12) it follows that

$$
\int d \mu \prod_{j=1}^{N}: e^{i \phi\left(f_{j}\right)}:=\exp \left[-\sum_{1 \leqq i<j \leqq N}\left\langle f_{i}, V f_{j}\right\rangle\right]
$$

By taking $f_{j}\left(\sigma^{\prime}, x^{\prime}\right) \rightarrow \sqrt{\beta} \delta\left(\sigma^{\prime}-\sigma_{j}\right) \delta\left(x^{\prime}-x_{j}\right)$ one concludes

$$
\int d \mu \prod_{j=1}^{N}: e^{i \sqrt{\beta} \phi\left(\sigma_{j}, x_{j}\right)}:=e^{-\beta U\left((\sigma)_{N},(x)_{N}\right)}
$$


We define

$$
C_{\Lambda}^{\beta}(\phi) \equiv \int_{S^{2}} d^{3} \sigma \int_{\Lambda} d^{3} x: \cos (\sqrt{\beta} \phi(\sigma, x)):
$$

From (13) the partition function can be written as

$$
\mathscr{Z}_{\Lambda}(\beta, z)=\int d \mu e^{z C_{\Lambda}^{\beta}}
$$

For $F(\phi) \in L^{1}(d \mu)$ we write

$$
\langle F\rangle_{\Lambda}(\beta, z)=\mathscr{Z}_{\Lambda}(\beta, z)^{-1} \int d \mu F(\phi) e^{z C_{\Lambda}^{\beta}}
$$

Using (13) one may check that

$$
\rho_{\Lambda}\left(\beta, z ; \alpha \sigma, x ; \alpha^{\prime} \sigma^{\prime}, x^{\prime}\right)=z^{2}\left\langle: e^{i \alpha \sqrt{\beta} \phi(\sigma, x)}:: e^{i \alpha^{\prime} \sqrt{\beta} \phi\left(\sigma^{\prime}, x\right)}\right\rangle_{\Lambda}(\beta, z)
$$

and so the effective potential can be written as

$$
W_{\Lambda}\left(\beta, z ; \alpha, x ; \alpha^{\prime}, x^{\prime}\right)=\left\langle\phi(\sigma, x) \phi\left(\sigma^{\prime}, x^{\prime}\right)\right\rangle_{\Lambda}(\beta, z)
$$

The above somewhat formal derivation can be justified rigorously [3].

We summarize some results in [3] for our system. In [3] we have shown that for $f \in \mathscr{S}\left(\mathbb{R}^{3} \times \mathbb{R}^{3}\right)$

$$
\left\langle e^{i \phi(f)}\right\rangle(\beta, z) \equiv \lim _{\Lambda \uparrow \mathbb{R}^{3}}\left\langle e^{i \phi(f)}\right\rangle_{\Lambda}(\beta, z)
$$

exists and it is the characteristic functional of a translation invariant probability measure on $\mathscr{S}^{\prime}\left(\mathbb{R}^{3} \times \mathbb{R}^{3}\right)$ (Theorem 4.2, [3]). Furthermore, the limit

$$
\langle\phi(\bar{f}) \phi(f)\rangle(\beta, z)=\lim _{\Lambda \uparrow \mathbb{R}^{3}}\langle\phi(\bar{f}) \phi(f)\rangle_{\Lambda}(\beta, z)
$$

exists and satisfies the bound (Theorem 4.3, [3]).

$$
\langle\phi(\bar{f}) \phi(f)\rangle(\beta, z) \leqq\langle f, V f\rangle
$$

for all $\beta$ and $z$. The above results are consequences of correlation inequalities [3] (also see [6]). We will need the following result:

Proposition 2. For any $f \in \mathscr{S}\left(\mathbb{R}^{3} \times \mathbb{R}^{3}\right)$

$$
\langle\phi(\bar{f}) \phi(f)\rangle(\beta, z) \geqq\left(1-(4 \pi / 3) z \beta e^{\beta K}\right)\langle f, V f\rangle
$$

where $K$ is defined in (10).

We postpone the proof of the proposition to the end of this note.

Using the translation invariance of the expectation $\langle>(\beta, z)$ we write

$$
\begin{aligned}
\langle\phi(\sigma, 0) \phi(\sigma, x)\rangle(\beta, z) & =\langle\phi(\sigma,-x / 2) \phi(\sigma, x / 2)\rangle(\beta, z) \\
& \equiv(2 \pi)^{-3 / 2} \int d^{3} k e^{i k \cdot x} \hat{W}(\sigma, \sigma ; k ; \beta, z)
\end{aligned}
$$

We write

$$
\begin{aligned}
\hat{V}(\sigma, \sigma ; k) & =(2 \pi)^{-3 / 2} \int d^{3} x e^{-k \cdot x} V(\sigma,-x / 2 ; \sigma, x / 2) \\
& =(\sigma \cdot k)^{2} k^{-2}|\hat{\kappa}(k)|^{2}
\end{aligned}
$$


Lemma 3. a) For $(4 \pi / 3) z \beta e^{K \beta}<1$ there exist a constant $a>0$ such that

$$
\begin{aligned}
& \hat{W}\left(\sigma, \sigma^{\prime} ; k ; \beta, z\right) \geqq a \hat{V}(\sigma, \sigma ; k) \\
& \text { b) For any } \beta \text { and } z \\
& \hat{W}(\sigma, \sigma ; k ; \beta, z) \leqq \hat{V}(\sigma, \sigma: k)
\end{aligned}
$$

Proof. By choosing a sequence of function $\left\{f_{n}\right\}, f_{n} \in \mathscr{S}$, such that

$$
\left|\hat{f}_{n}\left(\sigma^{\prime}, k^{\prime}\right)\right|^{2} \rightarrow \delta\left(\sigma^{\prime}-\sigma\right) \delta\left(k^{\prime}-k\right)
$$

the lemma follows from Proposition 2 and the bound (19).

Using Lemma 3 we now prove Theorem 1 . Without loss of generality we may choose $\sigma=e_{3}$, the unit vector along the positive $z$-axis. From (18) and (20) it follows that

$$
\begin{aligned}
W(x) & \equiv W\left(\beta, z ; e_{3},-x / 2 ; e_{3}, x / 2\right) \\
& =(2 \pi)^{-3 / 2} \int d^{3} k e^{i k \cdot x} \hat{W}(k) \\
\hat{W}(k) & =\hat{W}\left(e_{3}, e_{3} ; k ; \beta, z\right)
\end{aligned}
$$

Proof of Theorem 1. (a) We assume that

$$
\int|W(x)| d^{3} x<M
$$

for some $M<\infty$. By deducing a contradiction we prove the theorem. From Lemma 3 and (23) it follows that for $(4 \pi / 3) z \beta e^{K \beta}<1$ there exists a positive constant $a>0$ such that

$$
a \frac{k_{3}^{2}}{k^{2}}|\hat{\kappa}(k)|^{2} \leqq \hat{W}(k) \leqq \frac{k_{3}^{2}}{k^{2}}|\hat{\kappa}(k)|^{2}
$$

where $k=\left(k_{1}, k_{2}, k_{3}\right)$. We assert that $\hat{W}(k)$ is not continuous at the origin if $(4 \pi / 3) z \beta \cdot e^{K \beta}<1$. Choose a sequence $\left\{k^{(n)}\right\}, k^{(n)}=\left(\delta^{2 n}, \delta^{2 n}, \delta^{n}\right), 0<\delta<1$. As $n \rightarrow \infty$, $k^{(n)} \rightarrow(0,0,0)$. From the left inequality of $(24)$ and the fact that $\hat{\kappa}(0)=1$ it follows that $\lim _{n \rightarrow \infty} \hat{W}\left(k^{(n)}\right) \geqq a$. Next, choose a sequence $\left\{k^{(n)}\right\}, k^{(n)}=\left(\delta^{n}, \delta^{n}, \delta^{2 n}\right), 0<\delta<1$. From the right inequality $\lim _{n \rightarrow \infty} \hat{W}(k)=0$. This proves our assertion. We note that

$$
\hat{W}(k)-\hat{W}\left(k^{\prime}\right)=(2 \pi)^{-3 / 2} \int\left(e^{i k \cdot x}-e^{i k^{\prime} \cdot x}\right) W(x) d^{3} x
$$

By the assumption and the Lebesgue dominated convergence theorem we conclude that

$$
\hat{W}\left(k^{\prime}\right) \rightarrow \hat{W}(k) \quad \text { as } \quad k^{\prime} \rightarrow k \text { for all } k \in \mathbb{R}^{3}
$$

i.e. $\hat{W}(k)$ is continuous for all $k$. But this contradicts the previous deduction that $W(k)$ is discontinuous at the origin. This proves Theorem 1(a).

(b) Theorem 1(b) follows from the right inequality of (24). Thus we have proved Theorem 1 completely.

We now prove Proposition 2. 
Proof of Proposition 2. To show Proposition 2 we will use integration by parts [5]:

$$
\begin{gathered}
\int d \mu \phi(\sigma, x) F(\phi)=\int d \mu\left[\int d^{3} \sigma \int d^{3} x V\left(\sigma, x ; \sigma^{\prime}, x^{\prime}\right) \frac{\delta}{\delta \phi\left(\sigma^{\prime}, x^{\prime}\right)} F(\phi)\right] \\
\frac{\delta}{\delta \phi(\sigma, x)} \phi\left(\sigma^{\prime}, x^{\prime}\right)=\delta\left(\sigma-\sigma^{\prime}\right) \delta\left(x-x^{\prime}\right)
\end{gathered}
$$

The above formula is a consequence of the gaussian nature of the measure $d \mu(\phi)$ and it can be justified by finite approximation of $d \mu(\phi)$.

Remark. Alternatively one may derive the inequality (28) below directly (without using (25)) in the following way: expand $\exp \left[z C_{A}^{\beta}\right]$ by Taylor series in the both sides of (28) and compare the coefficients of $z^{N}$. Using the gaussian integration (13) one may compute the coefficients of $z^{N}$ of the both sides of (28) explicitly. These coefficients are

$$
\begin{aligned}
& \frac{1}{N !} \int d^{3} \sigma \int d^{3} x \int d^{3} \sigma^{\prime} \int d^{3} x^{\prime} \bar{f}(\sigma, x) f\left(\sigma^{\prime}, x^{\prime}\right) \\
& \quad \cdot \int_{\left(S^{2}\right)^{N}} d(\sigma)_{N} \int_{\Lambda^{N}} d(x)_{N} \bar{W}_{N}\left(\sigma, x ; \sigma^{\prime}, x^{\prime} ;(\sigma)_{N},(x)_{N}\right) e^{-\beta U\left((\sigma)_{N},(x)_{N}\right)}
\end{aligned}
$$

where

$$
\begin{aligned}
\bar{W}_{N}\left(\sigma, x ; \sigma^{\prime}, x^{\prime} ;(\sigma)_{N},(x)_{N}\right)= & V\left(\sigma, x ; \sigma^{\prime}, x^{\prime}\right) \\
& -\beta\left[\sum_{j=1}^{N} V\left(\sigma, x ; \sigma_{j}, x_{j}\right)\right]\left[\sum_{j=1}^{N} V\left(\sigma^{\prime}, x^{\prime} ; \sigma_{j}, x_{j}\right)\right]
\end{aligned}
$$

In this way one may prove the equality of (28) directly. In this sense we do not need (25) to show Theorem 1.

We now use (25) to derive (28) below. Define

$$
\begin{aligned}
A_{\Lambda}^{\beta}(\phi) & \equiv \iint d^{3} \sigma d^{3} x f(\sigma, x) V\left(\sigma, x ; \sigma^{\prime}, x^{\prime}\right) \frac{\delta}{\delta \phi\left(\sigma^{\prime}, x^{\prime}\right)} C_{\Lambda}^{\beta}(\phi) \\
& =-\sqrt{\beta} \int_{S^{2}} d^{3} \sigma \int_{\Lambda} d^{3} x(V f)(\sigma, x): \sin (\sqrt{\beta} \phi(\sigma, x)): \\
B_{\Lambda}^{\beta}(\phi) & =+\int d^{3} \sigma \int d^{3} x \bar{f}(\sigma, x) V\left(\sigma, x ; \sigma^{\prime}, x^{\prime}\right) \frac{\delta}{\delta \phi\left(\sigma^{\prime}, x^{\prime}\right)} A_{\Lambda}^{\beta}(\phi) \\
& =-\beta \int_{S^{2}} d^{3} \sigma \int_{\Lambda} d^{3} x|(V f)(\sigma, x)|^{2}: \cos (\sqrt{\beta} \phi(\sigma, x)):
\end{aligned}
$$

where

$$
(V f)(\sigma, x)=\int d^{3} \sigma^{\prime} \int d^{3} x^{\prime} V\left(\sigma, x ; \sigma^{\prime}, x^{\prime}\right) f\left(\sigma^{\prime}, x^{\prime}\right)
$$

From (25) and (26) we obtain

$$
\begin{aligned}
\int \phi(\bar{f}) \phi(f) e^{z C_{\Lambda}^{\beta}} d \mu= & \langle f, V f\rangle \mathscr{Z}_{\Lambda}(\beta, z) \\
& +z^{2} \int d \mu\left|A_{\Lambda}^{\beta}(\phi)\right|^{2} e^{z C_{\Lambda}^{\beta}}+z \int d \mu B_{\Lambda}^{\beta}(\phi) e^{z C_{\Lambda}^{\beta}}
\end{aligned}
$$


The second term of the right-hand side in (28) is positive. Since

$$
|: \cos (\sqrt{\beta} \phi(\sigma, x)):| \leqq e^{\beta K}
$$

where $K$ is defined in (10), we conclude that

$$
\langle\phi(\bar{f}) \phi(f)\rangle_{\Lambda}(\beta, z) \geqq\langle f, V f\rangle-z \beta e^{\beta K} \int_{S^{2}} d^{3} \sigma \int_{A} d^{3} x|(V f)(\sigma, x)|^{2}
$$

We write

$$
F(k)=\int d^{3} \sigma(\sigma \cdot k) \hat{f}(\sigma, k)
$$

We now use the definition of $V$ in (1) and the fact that $|\hat{\kappa}(k)| \leqq 1$ to obtain

$$
\begin{aligned}
\int_{S^{2}} d^{3} \sigma \int_{A} d^{3} x|(V f)(\sigma, x)|^{2} & \leqq \int_{S^{2}} d^{3} \sigma \int d^{3} x|(V f)(\sigma, x)|^{2} \\
& =\int_{S^{2}} d^{3} \sigma \int d^{3} k \frac{(\sigma \cdot k)^{2}}{k^{4}}|\hat{\kappa}(k)|^{2} \bar{F}(k) F(k) \\
& \leqq(4 \pi / 3) \int d^{3} k \frac{1}{k^{2}}|\hat{\kappa}(k)|^{2} \bar{F}(k) F(k) \\
& =(4 \pi / 3)\langle f, V f\rangle
\end{aligned}
$$

Using (30) and taking the limit $\Lambda \uparrow \mathbb{R}^{3}$ of (29) we complete the proof of Proposition 2.

Note. After the completion of this manuscript we learned that J. Fröhlich and T. Spencer have obtained a result similar to ours by a different method [8].

\section{References}

1. Brydges, D. : Commun. Math. Phys. 58, 313-350 (1978)

2. Fröhlich, J.: Commun. Math. Phys. 47, 233-268 (1976)

3. Fröhlich, J., Park, Y. M. : Commun. Math. Phys. 59, 235-266 (1978)

4. Fröhlich, J., Park, Y. M. : In preparation

5. Glimm, J., Jaffe, A., Spencer, T. : The contribution in constructive quantum field theory (eds. G. Velo, A. Wightman). Lecture notes in physics, Vol. 25. Berlin, Heidelberg, New York : Springer 1973

6. Park, Y. M.: J. Math. Phys. 18, 2423-2426 (1977)

7. Ruelle, D.: Statistical mechanics. Reading-London-Amsterdam-Tokyo: Benjamin 1969

8. Fröhlich, J., Spencer, T. : Private communication

Communicated by E. Lieb

Received June 18, 1979 
